\title{
The Effects of Spatial and Temporal Grids on Simulations of Thin Films with Surfactant
}

\author{
Celeste Conti \\ Harvey Mudd College \\ (916) 704-7209 \\ celeste_conti@hmc.edu
}

\author{
Eric A. Autry \\ Harvey Mudd College \\ (515) 314-3013 \\ eric_autry@hmc.edu \\ Rachel Levy \\ Faculty Advisor \\ Harvey Mudd College \\ Department of Mathematics \\ 301 Platt Blvd. \\ Claremont, CA 91711 \\ (909) 607-6019
}

\author{
Greg Kronmiller \\ Harvey Mudd College \\ (919) 257-3617 \\ greg_kronmiller@hmc.edu
}

April 8, 2013

\begin{abstract}
In this work, we investigated a numerical solver that combines Alternating Direction Implicit (ADI) methods with CLAWPACK to address mixed-type equations, such as the parabolic-hyperbolic system of PDEs describing surfactant spreading on a thin liquid film. In particular, we probed the effects of the spatial and temporal grid on the results of the simulations. Spatial grid effects were studied by rotating a controlled set of initial conditions relative to the grid, while temporal grid effects were studied by varying the time step and spatial resolution.
\end{abstract}

\section{Introduction}

Claridge, Levy, and Wong [2] have developed an open source, first order numerical solver that simulates the evolution of a thin liquid film. We demonstrate the effectiveness of this code in solving a system of coupled nonlinear partial differential equations that models the evolution of a thin liquid film with insoluble surfactant. These equations are of mixed type, both hyperbolic and parabolic. The novelty of this code is that it solves hyperbolic and parabolic terms of the model separately to accurately resolve features of the solution characteristic of each type of equation.

The purpose of this investigation is to quantify the effects of the rectangular spatial grid on the results of these simulations. Specifically, we discuss how the symmetry of a solution depends on the spatial resolution, as well as the relationship between the spatial resolution of a simulation and the time step required for that simulation to run most efficiently. The results of these investigations may benefit future users of this numerical solver by informing their choice of spatial and temporal resolution; with these findings, users may choose a spatial resolution that reduces asymmetry to a tolerable level and a time step that allows the simulation to run as fast as possible given the chosen spatial resolution. 


\subsection{The Model}

The numerical solver employed in this paper solves thin film equations, such as the system of nonlinear partial differential equations modeling a thin film with insoluble surfactant:

$$
\begin{gathered}
h_{t}+\nabla \cdot\left(\frac{1}{2} h^{2} \nabla \sigma\right)=\beta \nabla \cdot\left(\frac{1}{3} h^{3} \nabla h\right)-\kappa \nabla \cdot\left(\frac{1}{3} h^{3} \nabla \nabla^{2} h\right) \\
\Gamma_{t}+\nabla \cdot(h \Gamma \nabla \sigma)=\beta \nabla \cdot\left(\frac{1}{2} h^{2} \Gamma \nabla h\right)-\kappa \nabla \cdot\left(\frac{1}{2} h^{2} \Gamma \nabla \nabla^{2} h\right)+\delta \nabla^{2} \Gamma .
\end{gathered}
$$

Eq. 1a models the evolution of the film height, $h(x, y, t)$, and Eq. 1b models the surfactant concentration, $\Gamma(x, y, t)$, where $x$ and $y$ are the non-dimensionalized spatial coordinates and $t$ is non-dimensionalized time. In both equations, the $\nabla \sigma$ term describes the Marangoni forces caused by the surfactant; this is what drives the system. The function $\sigma(\Gamma)=(1+\mu \Gamma)^{-3}$ [1] is the equation of state, which describes the effect of the surfactant on the surface tension of the film. The non-dimensionalized coefficients, $\beta, \kappa$, and $\delta$, describe the relative strengths of the various forces acting on the film. The second-order $\beta$ terms describe the effects of gravity in the spreading of the film and the surfactant, while the fourth-order $\kappa$ terms describe the effects of capillarity on the system. The surfactant equation also has a second-order $\delta$ term, which describes the diffusion of the surfactant across the surface of the film. The non-dimensionalized coefficients can be expressed as

$$
\beta=\frac{\rho g H^{2}}{S}, \quad \kappa=\frac{\gamma H^{2}}{S L^{2}}, \quad \delta=\frac{\mu D}{S H},
$$

where $\rho$ is the fluid density, $\mu$ is the fluid viscosity, $\gamma$ is the surface tension parameter, $D$ is the surface diffusivity, $g$ is the acceleration due to gravity, $L$ is the radius of the ring, $H$ is the initial film height, and $S$ is the reduced surface tension, or the difference between the surface tension of glycerin and the surface tension of glycerin with a monolayer of surfactant.

The terms in these equations can be classified as either parabolic or hyperbolic based on the highest order derivative in each term. In the film height equation, Eq. 1a, the $\beta$ and $\kappa$ terms each contain a second derivative of $h$. Similarly, in the surfactant equation, Eq. $1 \mathrm{~b}$, the $\nabla \sigma$ and the $\delta$ terms contain a second derivative of $\Gamma$. Therefore, these terms behave similarly to the heat equation and are classified as parabolic. In contrast, the $\nabla \sigma$ term in Eq. 1a contains a first derivative of $h$, and the $\beta$ and $\kappa$ terms in Eq. 1b contain first derivatives of $\Gamma$. Therefore, these terms behave similarly to the wave equation and are classified as hyperbolic.

We chose to use this system of equations to study the effects of the spatial grid on the symmetry of a solution. In addition, we explored the relationship between the time step and the spatial resolution of a simulation. Our goal was to understand how we could limit the effects of the grid size on the result of a simulation while maintaining a reasonable run time.

\subsection{The Numerical Solver}

The numerical solver developed by Claridge, Levy, and Wong is designed to solve nonlinear systems of partial differential equations involving both parabolic and hyperbolic terms. It is based on a finite volume method, where the surfactant concentration and film height are averaged over each cell, yielding the values associated with the cell center. The hyperbolic terms in the system are solved using CLAWPACK [5]. The parabolic terms are solved using an Alternating Direction Implicit (ADI) method. These simulations used a no-flux boundary condition. We performed a grid refinement test to confirm that this code is first order.

\section{Rotation and Grid Effects}

In an ideal numerical simulation, the orientation of a symmetric initial condition with respect to the spatial grid would not affect the results of a simulation. However, when a rectangular grid is imposed on a symmetric 
initial condition such that the grid doesn't align with the axis of symmetry, we found that the grid introduces small asymmetries ${ }^{1}$ into the simulation. Understanding these numerical errors and how to minimize them is important if we want to compare the results of these simulations to experimental results; in order to make meaningful comparisons, we must quantify the uncertainty that arises from spatial and temporal discretizations.

We developed two methods of measuring the error associated with misalignment on a rectangular grid. The first method explores how rotating an initial condition by an angle $\theta$ affects the symmetry of corresponding points across the visual line of symmetry after running the simulation; this method measures how much asymmetry is introduced by the rotation in a single simulation. The second method compares the results of two simulations that differ only by the rotation angle $\theta$ of the initial condition; this comparison indicates how much the solutions vary based on the alignment of the initial condition to the spatial grid.

\subsection{General Initial Condition}

In this section, we rotated an initial condition and observed how the simulation progressed differently due to that rotation. In recent experiments, such as those described in [3], the surfactant is generally deposited on the film in a shape that is rotationally symmetric with respect to every angle. However, such an initial condition is not suitable for this study because rotating it has no effect. Therefore, to probe the effects of rotation on a simulation, we needed an initial condition with limited rotational symmetry.

For both methods, we chose a film of uniform height, $h(x, y, 0)=1$, and a non-zero initial surfactant concentration inside a plus-shaped region. The four arms of the plus sign each have length 1 and width 0.5 , and the initial condition is rotated counterclockwise from alignment with the grid by an angle $\theta$.

The choice of shape for the surfactant concentration profile is motivated by simulations in [6], which tracked the speed and location of the leading edge of the surfactant. To be consistent with this work, we have defined a continuous initial surfactant concentration that is non-differentiable at the boundaries of the plus shape.

The initial surfactant concentration is calculated as follows. Let the $u$ - and $v$-axes be aligned with the plus sign (i.e. the $x$ - and $y$-axes (respectively) rotated counterclockwise by the angle $\theta$ ), and let $(u, v)$ be the coordinates of some point inside the plus sign with respect to the $u$ - and $v$-axes (Fig. 1). Note that $(u, v)$ can be found by rotating the point $(x, y)$ clockwise by the angle $\theta$. Then define the surfactant concentration to be:

$$
\Gamma(u, v)= \begin{cases}\left(1-\left(\frac{\max (|u|,|v|)}{1.0}\right)^{10}\right)\left(1-\left(\frac{\min (|u|,|v|)}{0.25}\right)^{10}\right) & \text { if } \max (|u|,|v|)<1.0 \text { and } \min (|u|,|v|)<0.25 \\ 0 & \text { otherwise }\end{cases}
$$

The 1.0 and 0.25 in the denominators above are, respectively, the length and half the width of each arm of the plus sign. The surfactant concentration at the boundaries of the plus sign (where $\max (|u|,|v|)=1.0$ or $\min (|u|,|v|)=0.25)$ is zero, and as you move away from the boundary into the plus sign, the surfactant concentration rapidly rises toward 1. A surface plot of this equation is shown in Fig. 2. Additionally, by taking the absolute values of $u$ and $v$ and using their maximum and minimum values rather than using $u$ and $v$ directly, we construct the plus sign in an inherently symmetric way. The surfactant concentration is computed at discrete points (the centers of the cells) for one-eighth of the plus sign's area (Fig. 3), and the remaining values are found by reflecting and rotating this region. An example of how this initial condition evolves in time is shown in Fig. 4.

\subsection{Measurement 1: Asymmetry of the Solution}

To probe the effects of the rectangular spatial grid, we compared several simulations using the rotated plus sign initial condition described above in Section 2.1. We varied two parameters, the distance between cell centers, $\Delta x$, and the angle of rotation, $\theta$.

\footnotetext{
${ }^{1}$ In general, these asymmetries are not easily visible unless the spatial discretization is very coarse. However, we show that these asymmetries can introduce significant error in Section 2.4 .
} 


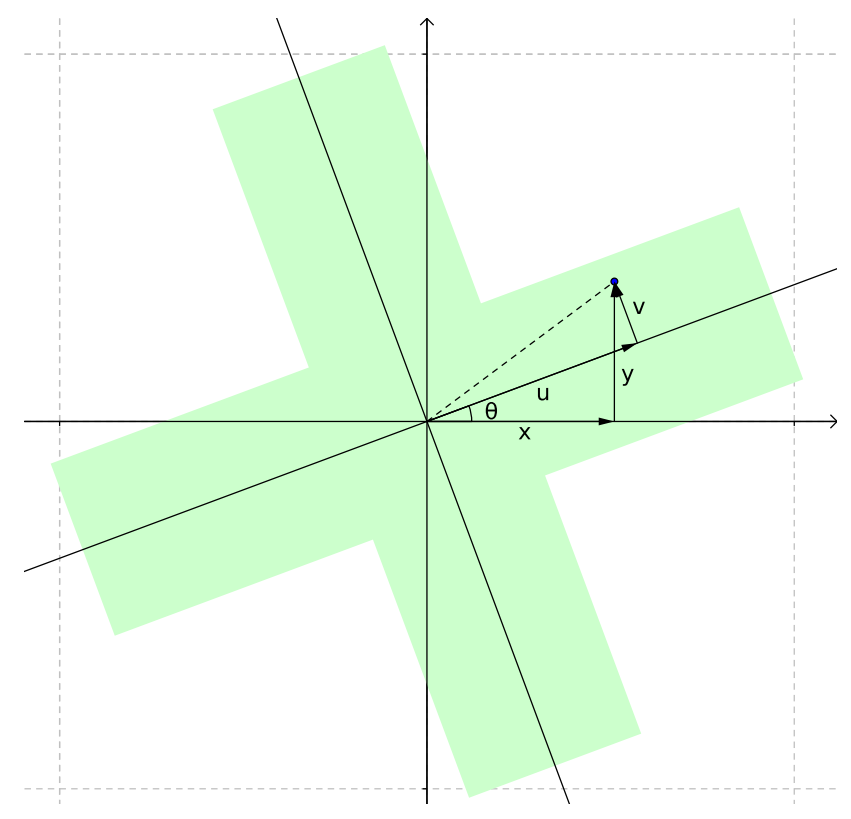

Figure 1: The rotated plus sign initial condition. The shaded region contains surfactant, and the points $(x, y)$ and $(u, v)$ are representative of each coordinate system. The gray dashed lines on the perimeter are reference lines located at $x= \pm 1$ and $y= \pm 1$.

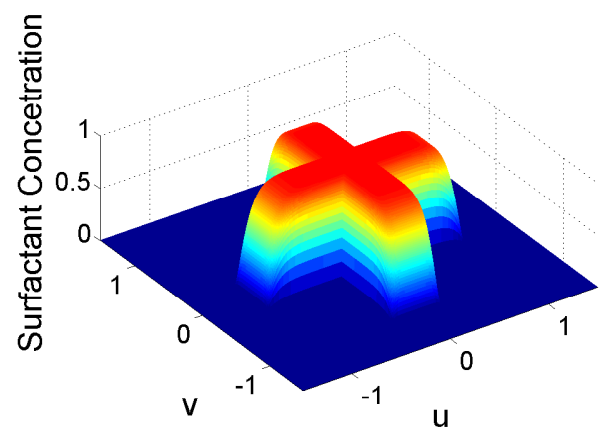

Figure 2: A surface plot showing the surfactant concentration (in number of monolayers) as a function of position for the rotated plus sign initial condition.

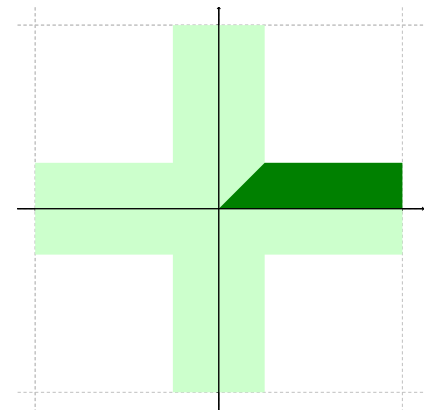

Figure 3: The unrotated plus sign initial condition. The darker region shows where $\max (|u|,|v|)=x$ and $\min (|u|,|v|)=y$. Surfactant concentration on the rest of the plus sign is defined by rotating and reflecting the values in the darker region. 


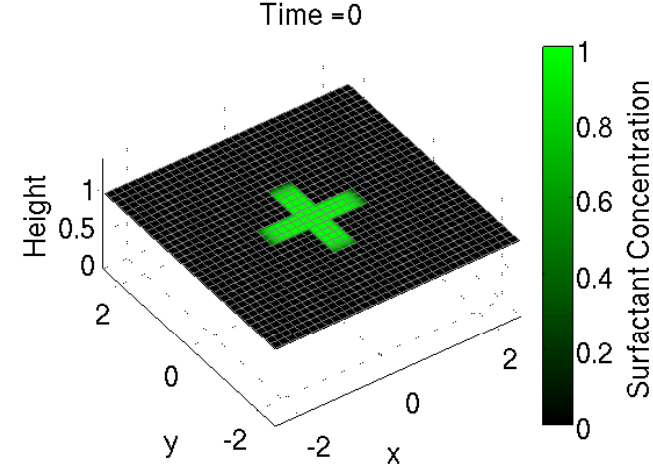

(a)

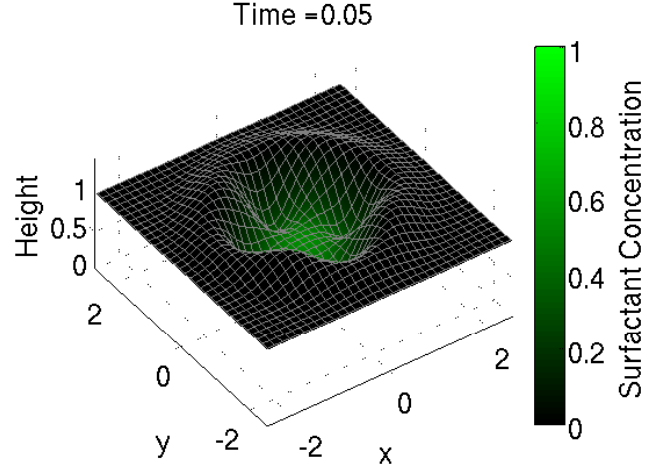

(b)

Figure 4: The plus-sign initial condition and a later frame showing how the system evolves in time. The height of the film is plotted on the $z$-axis, and the concentration of surfactant is represented by the color of the surface.

We developed two metrics to measure the asymmetry of a solution across its expected axis of symmetry. Both metrics compare cells at corresponding locations across an axis of symmetry of the initial condition. The first is the Average Squared Difference metric, which does not depend on the grid spacing but is strongly affected by the size of the domain relative to the initial surface area containing surfactant. The second is the Maximum Difference metric, which is sensitive to outliers but is easier to compare across simulations.

In describing these metrics, we use the following naming conventions. Points in the domain are referred to by their $x$ and $y$ coordinates. The spatial domain is divided into rectangular cells, which are indexed by $i$ in the $x$ direction and by $j$ in the $y$ direction, where $i$ ranges from $1,2, \ldots, i_{\text {max }}$ and $j$ ranges from $1,2, \ldots, j_{\max }$. The choice of $i_{\max }$ and $j_{\max }$ combined with the size of the domain determines the spatial resolution of the simulation, or the number of cells per unit length in the $x$ and $y$ directions. Recall that the finite volume method computes cell averages, which are then associated with the center of each cell.

Average Squared Difference The first metric of asymmetry was calculated as follows:

1. For each cell, reflect the coordinates of its center across an axis of symmetry of the initial condition. Note that the axis of symmetry relates $x$ and $y$ coordinates, not $i$ and $j$ indices. (Some points will reflect to coordinates outside of the computational domain. These points are excluded from the calculation.)

2. In general, the reflected coordinates will not correspond to the exact center of a cell. Use a bilinear interpolation $^{2}$ on the four cell centers nearest to the reflected point to generate a single value for comparison. This value approximates the value at the reflected coordinates, so if the solution is symmetric, then this value should be close to the value of the cell that was reflected.

3. Square the difference between the interpolated value and the value associated with the cell that was reflected. Since the interpolated value doesn't correspond to an actual cell, we do not expect this value to be zero; however, because the film height and surfactant concentration are expected to be continuous, it should be small. ${ }^{3}$

\footnotetext{
${ }^{2} \mathrm{~A}$ linear interpolation weights points according to their proximity to the coordinate of interest. A bilinear interpolation performs two pairwise interpolations and then interpolates these values.

${ }^{3}$ It should be noted that since we are comparing the value at the center of a cell on one side of the expected axis of symmetry with a weighted average of four cell centers on the other side, the two values compared will not match exactly even if the simulation is perfectly symmetric. However, since we use a continuous function for the surfactant profile, this difference should be small, especially at finer spatial resolutions. Nonetheless, this method will tend to overestimate the asymmetry of the simulation. This means that our measurements of asymmetry, rather than being a precise measure of how asymmetric the plot is, instead represent an upper bound on the asymmetry.
} 
4. Calculate the average of the squared differences over all cells. This value provides a measure of asymmetry.

We use an average so that the result will not depend on the resolution of the grid. It is difficult to quantify the dependence of the Average Squared Difference metric on the relative sizes of the domain and the region containing surfactant. To interpret an asymmetry value, we must compare it to other asymmetry values for simulations using the same domain size; a single asymmetry value is nearly impossible to interpret without these comparisons. The limitations of the Average Squared Difference metric motivated us to investigate an alternate method that provides an easier-to-interpret asymmetry value.

Maximum Difference The Maximum Difference metric is calculated by reflecting the coordinates of each cell across the visual line of symmetry and interpolating, as before. For the Maximum Difference metric, however, instead of summing squared differences, we simply track the largest difference. The advantage of this metric is that the units of the asymmetry measurements are more intuitive; they are simply a fraction of the characteristic height. For example, if the maximum difference is on the order of 0.01 times the characteristic height, then the worst asymmetry error is only a $1 \%$ difference, which is acceptable for first order code. However, the disadvantage of the Maximum Difference metric is that it is sensitive to outliers; one large outlier will obscure the rest of the data.

We chose to use the Maximum Difference metric of asymmetry; for the remainder of this paper, measures of asymmetry are made using this metric.

\subsection{Measurement 2: Rotation Effects}

Method 1 measures the asymmetry introduced when the axis of symmetry of the initial condition is not aligned with the spatial grid. However, it is possible that if we compare two simulations whose initial conditions only differ by an angle of rotation, we will find that the solutions differ from each other even though they are both symmetric; in other words, symmetry does not guarantee that the solution of a simulation with a rotated initial condition will yield the same results as a simulation whose axis of symmetry is aligned with the grid. We use the term rotational mismatch to refer to the possible differences between solutions whose initial conditions only differ by an angle of rotation.

Whereas in Method 1 we compared corresponding points across the visual line of symmetry, in Method 2 we quantify the rotational mismatch by comparing the results of two simulations whose initial conditions differ only by the angle of rotation, $\theta$. The unrotated initial condition has an axis of symmetry aligned with the spatial grid. To compare the two simulations, we need to de-rotate the result of a rotated simulation and compare the result to a simulation that was not rotated in the first place. The de-rotated plot is generated and the comparison is made as follows:

1. Create a new array, of the same size as the other plots, with uninitialized cell values. This will become the de-rotated plot.

2. Calculate cell values in the new plot as follows:

(a) Rotate the coordinates of a cell from the new plot counterclockwise about the origin by the angle $\theta$. Use domain coordinates $(x, y)$, not cell indices $(i, j)$.

(b) Compute a bilinear interpolation of the values at the four cell centers nearest these coordinates in the rotated simulation. This provides the value of the cell in the new plot. (If the rotated coordinates are outside of the domain, omit this point from the analysis.)

3. This process yields a plot of the rotated simulation de-rotated, so that its expected axis of symmetry is aligned with the grid again.

4. Compare corresponding cells in the de-rotated plot and the plot from the unrotated run. We chose to use the Maximum Difference metric. 
The comparison between the de-rotated plot and the plot from the unrotated run is intended to measure the rotational mismatch. However, it is important to note that some additional error may be introduced by the bilinear interpolation used to generate the de-rotated plot. Therefore, the value produced by this method is only an upper bound for the rotational mismatch.

\subsection{Symmetry Results}

Using the two metrics defined in the previous sections, we can now determine the conditions necessary to reduce grid effects to tolerable levels. In this section, we examine how the asymmetry of a simulation changes over time, as well as how the asymmetry and the rotational mismatch depend on the angle of rotation. All simulations use the rotated plus sign initial condition described in Section 2.1. We vary two parameters, the distance between cell centers, $\Delta x$, and the angle of rotation, $\theta$. Each simulation has a square domain with a side of length 8. The square grid cells of each simulation have a length of $\Delta x=0.1$ (coarse resolution), $\Delta x=0.05$, or $\Delta x=0.025$ (fine resolution). The angle of rotation of the initial condition range from $0^{\circ}$ to $90^{\circ}$ by intervals of $11.25^{\circ}$ (giving nine angles of rotation).

In all of our simulations, we show that the asymmetry in both the film height and the surfactant concentration decrease as the simulation progresses. The least asymmetry occurs for angles of rotation that are more closely aligned with the grid. As expected, simulations run at an angle of $0^{\circ}, 45^{\circ}$, and $90^{\circ}$ have virtually no asymmetry. However, there is significant rotational mismatch between simulations run at $45^{\circ}$ and those run at $0^{\circ}\left(\right.$ or $\left.90^{\circ}\right)$. The asymmetry and the rotational mismatch both decrease as the spatial grid is refined; a grid size of $\Delta x=0.05$ is sufficient to limit both of these errors to less than $1 \%$ in all simulations.

Let us now discuss the simulations in greater detail. The first question we investigated was whether or not asymmetries in the initial condition would persist during the simulation. Fig. 5 shows asymmetry in height versus time for a resolution of $\Delta x=0.025$. The data for corresponding angles of rotation $\left(0^{\circ}\right.$ and $90^{\circ} ; 11.25^{\circ}$ and $78.75^{\circ} ; 22.5^{\circ}$ and $67.5^{\circ}$; and $33.75^{\circ}$ and $56.25^{\circ}$ ) are identical to within $0.1 \%$ error, which is expected since such runs are equally well aligned with the grid; the points plotted in Fig. 5 represent the average of the data from each corresponding pair of angles. The simulations with initial conditions rotated $0^{\circ}, 45^{\circ}$, or $90^{\circ}$ evolved symmetrically, which is unsurprising since these initial conditions are aligned with the grid. The remaining data series for corresponding angles have the same general pattern: they increase to a local maximum, then decrease over time. Since the initial height is uniform (and therefore perfectly symmetric), the initial increase is to be expected. The subsequent local max followed by a decrease is encouraging; it implies any asymmetries in the initial condition tend to smooth away in time rather than propagating through the simulation. ${ }^{4}$

Similarly, we found that the asymmetry in the surfactant concentration also decreases with time. The plot of surfactant concentration asymmetry versus time (Fig. 6) shows that surfactant concentration is most asymmetric at $t=0$, then quickly approaches 0 ; again, this implies that the asymmetry does not propagate as the simulation progresses. The smoothing terms in Eq.1a and Eq.1b probably cause the sudden decrease in asymmetry. However, it is possible that the solution would remain asymmetric if there were no smoothing terms in the equations. The inset in Fig. 6 is rescaled to better show the data after time 0 .

Once we established that asymmetries do not persist through a simulation, we wanted to know how the angle of rotation and the resolution of the spatial grid affect the asymmetry. Fig. 7 shows the asymmetry in film height versus angle at various spatial resolutions. The corresponding graph for surfactant concentration has a very similar shape and so has been omitted. As mentioned above, for initial conditions rotated by $0^{\circ}, 45^{\circ}$, or $90^{\circ}$, the plus sign is aligned with the grid and the solution is therefore quite symmetric. The asymmetry increases as the angle of rotation moves further away from one of these three angles. For all angles, the asymmetry decreases as the resolution increases (i.e., as $\Delta x$ decreases). Since the asymmetry decreases as the grid is refined regardless of the angle of rotation, we know that for any acceptable level of asymmetry, we can choose a sufficiently small spatial resolution to ensure that our asymmetry does not exceed this level. For instance, given this particular initial condition, we can say that the asymmetry in film

\footnotetext{
${ }^{4} \mathrm{On}$ a domain of the chosen size, waves created by the parabolic terms have reached the edges of the domain by time 1.3 . After this time, asymmetry increases once more as the waves reflect from the boundary. However, this rise in asymmetry is not present (or is delayed) in simulations with larger domains.
} 

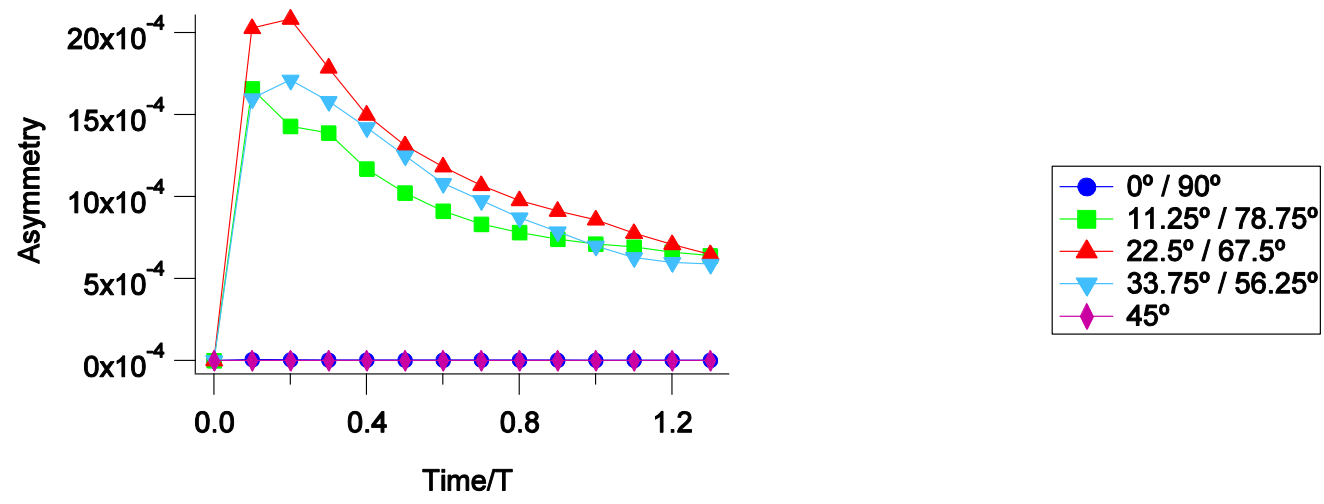

Figure 5: Asymmetry in height over time at various angles of rotation and a resolution of $\Delta x=0.025$ measured with the Maximum Difference metric described in Section 2.2.
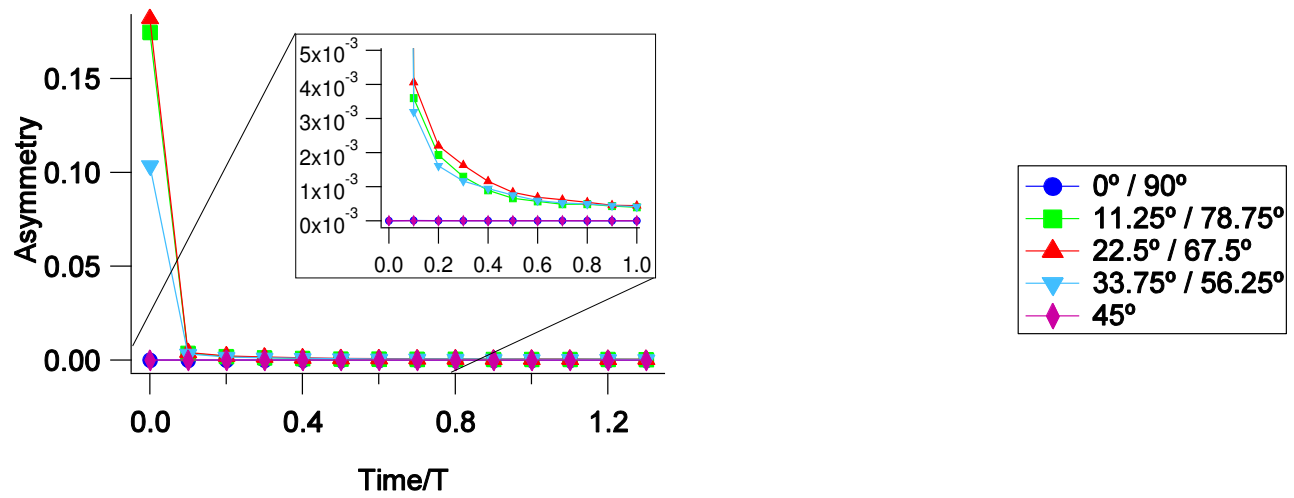

Figure 6: Asymmetry in surfactant concentration over time at various angles of rotation and a resolution of $\Delta x=0.025$, measured with the Maximum Difference metric. 
height is never worse than $1 \%$ of the characteristic height for $\Delta x \leq 0.05$. The asymmetries for surfactant concentration are actually slightly higher; if we require at most a $1 \%$ error in surfactant concentration, the necessary spatial resolution may be as small as $\Delta x=0.025$.
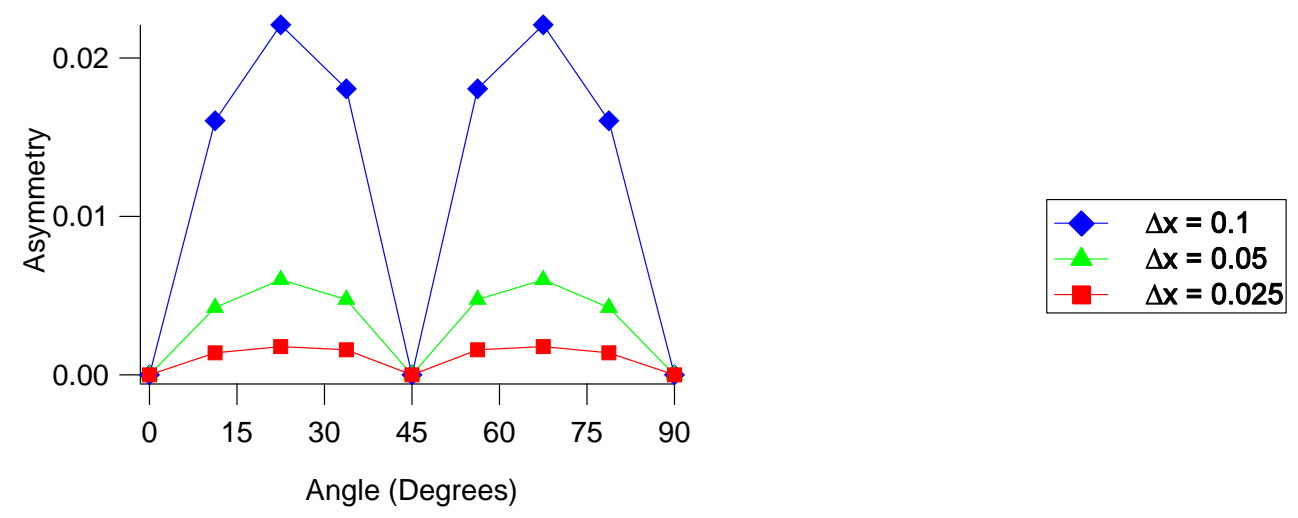

Figure 7: Asymmetry in height versus angle of rotation at various spatial resolutions and at time 0.3 measured with the Maximum Difference metric. The shape is reasonable: low asymmetry at $0^{\circ}, 45^{\circ}$, and $90^{\circ}$, and high in between.

As discussed previously in Section 2.3, it is possible for a solution to be symmetric but still differ from the result of a simulation with the same initial condition rotated by a different angle. Recall that we define rotational mismatch to be the difference between the results of two such simulations. After determining that it is possible to limit asymmetry by refining the spatial grid, we investigated how the rotational mismatch of a simulation depended on the angle of rotation and the size of the spatial grid. A plot of rotational mismatch of the film height versus angle of rotation for various spatial resolutions is shown in Fig. 8. Again, rotational mismatch decreases as the resolution is increased. In fact, the shape is somewhat reminiscent of the analogous asymmetry graph, but with one important difference. While simulations with an initial condition rotated at $45^{\circ}$ have virtually no asymmetry, they have the largest rotational mismatch. This implies that a $45^{\circ}$-rotated plus sign evolves very symmetrically, but quite differently from an unrotated plus sign. Because rotational mismatch decreases for all angles of rotation as the grid is refined, we can again choose a sufficiently large spatial resolution to ensure that the rotational mismatch is less than any given value. In this case, we can reduce the rotational mismatch to less than $1 \%$ using a spatial grid of size $\Delta x=0.05 .{ }^{5}$ Because of errors introduced in making the de-rotated plot, as previously mentioned, this is actually an upper bound on the error. These answers are encouraging; a $\Delta x$ of 0.05 is reasonably coarse, yet seems to be sufficient to substantially limit grid effects.

\section{Time Step and Resolution}

For each simulation, the user must choose a spatial resolution and provide an initial time step for the solver. Both the CLAWPACK and ADI portions of the code use adaptive time stepping to improve runtime; however, the user must give a reasonable initial time step in order for the simulation to converge to a solution. Too large an initial time step will cause the simulation to fail, either because the Courant-Friedrichs-Lewy (CFL) condition [4] is not met or because Newton's method fails to converge; too small an initial time step will cause the simulation to run slowly. Our goal was to describe the relationship between the largest possible (and therefore most efficient) time step and the grid size of a particular run.

\footnotetext{
${ }^{5} \mathrm{As}$ in the results for asymmetry, the rotational mismatch error in the surfactant concentration is slightly higher than the rotational mismatch error in the film height. To limit rotational mismatch error to less than $1 \%$ in both film height and surfactant concentration, we need $\Delta x$ between 0.05 and 0.025 .
} 


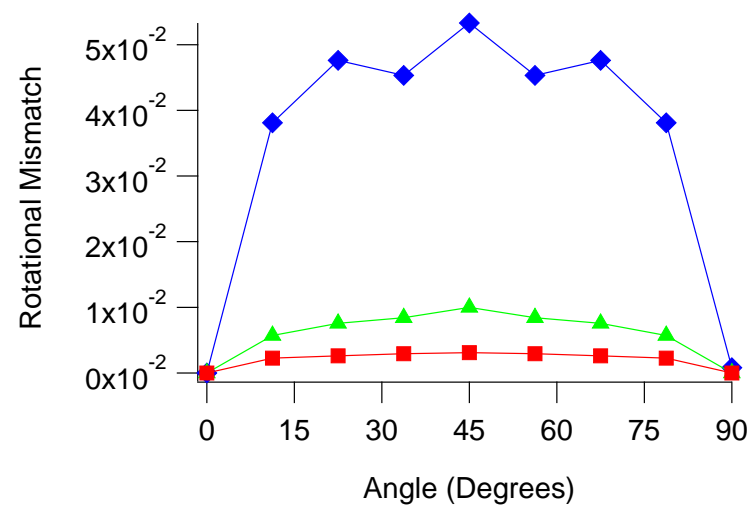

$\Delta x=0.1$

$\Delta \mathrm{x}=0.05$

$\Delta \mathrm{x}=0.025$

Figure 8: Rotational mismatch in height versus angle of rotation at various spatial resolutions and at time 0.3 measured using the Maximum Difference metric.

\subsection{Temporal Grid Refinement}

We noted that in general, if a run failed (either because the CFL condition was not met or because Newton's method failed to converge ${ }^{6}$ ) it would do so within the first 0.5 non-dimensionalized time units. Therefore, for the purposes of this investigation, we define a successful run to be one that reached 1.8 non-dimensionalized time units, where 1.8 was chosen for its divisibility properties. While 1.8 time units may sound small, in a typical simulation, a disk-shaped surfactant initial condition could easily have doubled in diameter in this time.

To study the relationship between the size of the grid cells, $\Delta x$, and the time step, $\Delta t$, we ran several simulations with identical initial conditions, varying only the time step or the grid size, and recorded which runs were successful. For these runs, the adaptive time stepping feature was disabled in both the CLAWPACK and ADI portions of the code, which ensured that the reported time step worked for the entire simulation. However, in practice users will take advantage of the variable time step feature, and a larger initial time step could be used.

To study the effects of the spatial and temporal resolution on a simulation, we chose to use an outwardspreading disk initial condition ${ }^{7}$. This choice was motivated by experiments being performed at the Daniels Lab at North Carolina State University [3]. In these experiments, a metal cylinder is placed in the middle of a thin film of $99.5 \%$ anhydrous glycerin, and a monolayer of surfactant (a fluorescently tagged lipid) is placed inside the cylinder. When the cylinder is lifted from the film, the surfactant begins to spread outward, and changes in film height and surfactant concentration are measured using a laser line and a fluorescencetracking camera. Because in this section we are not studying the effects of rotation, we can choose an initial condition that more closely models these experiments.

We chose to define the outward-spreading disk initial condition in two ways. In both cases, the height of the film was uniformly $h=1$, and the surfactant was contained in a disk of radius 1 . The surfactant is assumed to not add to the height of the film; a higher concentration means that the monolayer of molecules is more densely packed. The surfactant concentration profile was defined in two ways. The first was continuous

\footnotetext{
${ }^{6}$ In some cases, the simulation did not fail in the sense that the CFL condition was satisfied and Newton's method was able to converge, but the results of the simulation were nonsensical; for instance, with very coarse grids (on the order of $\Delta x=0.2$ ), we noted negative values for the film height and surfactant concentration. These runs are considered to have failed.

${ }^{7}$ In general, we found that the time step and grid size required for a particular simulation depended more on the derivate of the initial surfactant concentration (i.e. the difference in surfactant concentration between adjacent cells) than on the specific shape of the region containing surfactant. Therefore, we expect the results from this section to apply to initial conditions of any shape, including the rotated plus sign defined in Section 2.1
} 


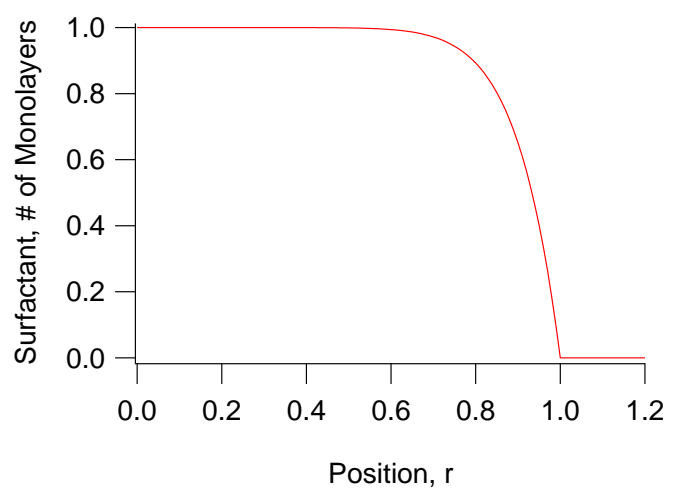

(a) Surfactant concentration in number of monolayers as a function of the radial position, Eq. 3a.

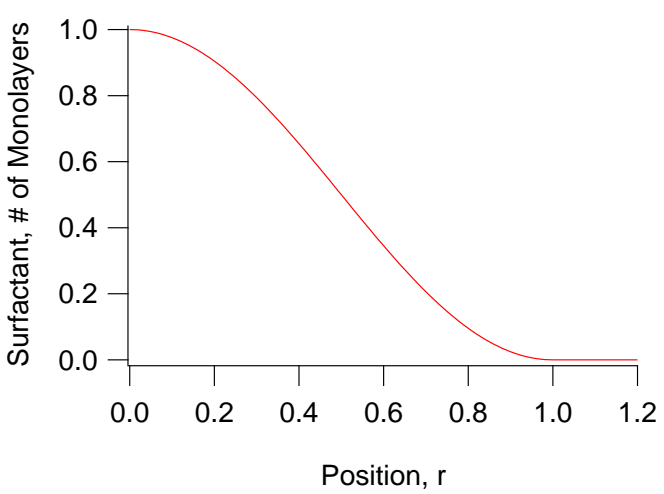

(b) Surfactant concentration in number of monolayers as a function of the radial position, Eq. $3 \mathrm{~b}$.

Figure 9: Surfactant concentration profiles for the two initial conditions used in the temporal grid refinement analysis. Note that Eq. 3a is not differentiable at $r=1$ (Fig. 9(a)), while Eq. 3b is continuously differentiable (Fig. 9(b)).

everywhere, but was not differentiable at the boundary of the disk:

$$
\Gamma(r)= \begin{cases}\left(1-r^{10}\right) & \text { for } r \leq 1 \\ 0 & \text { elsewhere }\end{cases}
$$

The second was differentiable everywhere:

$$
\Gamma(r)= \begin{cases}\frac{1}{2}(\cos (\pi r)+1) & \text { for } r \leq 1 \\ 0 & \text { elsewhere }\end{cases}
$$

In both cases, the surfactant concentration is defined in polar coordinates, i.e., $r=\sqrt{x^{2}+y^{2}}$. (Note that the simulations are still two dimensional; we have simply defined the initial surfactant condition in an axisymmetric way.) A plot of each of these functions is included in Fig. 9. The motivation for the corner in the first profile, as with the plus sign initial condition, is to be consistent with initial conditions used in [6]. Both initial conditions are used in the following temporal grid refinement study.

Fig. 10 summarizes the results of the simulations of outward spreading disks, where the time step and the spatial resolution were varied. It shows the time step, $\Delta t$, vs. the grid size, $\Delta x$, on a log-log scale. Each error bar represents the range in which we know the largest possible time step for a given spatial resolution exists; runs with time steps larger than the upper edge of the error bar will fail, while runs with time steps smaller than the lower edge of the error bar will succeed. It is possible to shrink the error bar further by running additional simulations with intermediate values for the time step. The point on the graph is the average of the upper and lower edges of the error bar and does not represent an actual simulation. The equation for a power law was fit to this data, and the relationship between the maximum time step and the spatial resolution is at worst approximately a power law of the form $\Delta t \propto \Delta x^{4.5}$.

It is worth noting that the relationship between the time step and the grid size is more closely a power law for the differentiable outward-spreading disk initial condition than for the non-differentiable outwardspreading disk initial condition. As the spatial resolution is increased, the time steps required by each initial condition seem to converge to a common curve. This suggests that smaller time steps are required when there are larger differences in surfactant concentration between adjacent cells. As the grid is refined, the difference in concentration between adjacent cells is decreased, so that the time step required by the differentiable and non-differentiable initial conditions become more similar. This implies that the smallest time steps are required at the very beginning of the simulation; once the system has started to evolve and the surfactant 

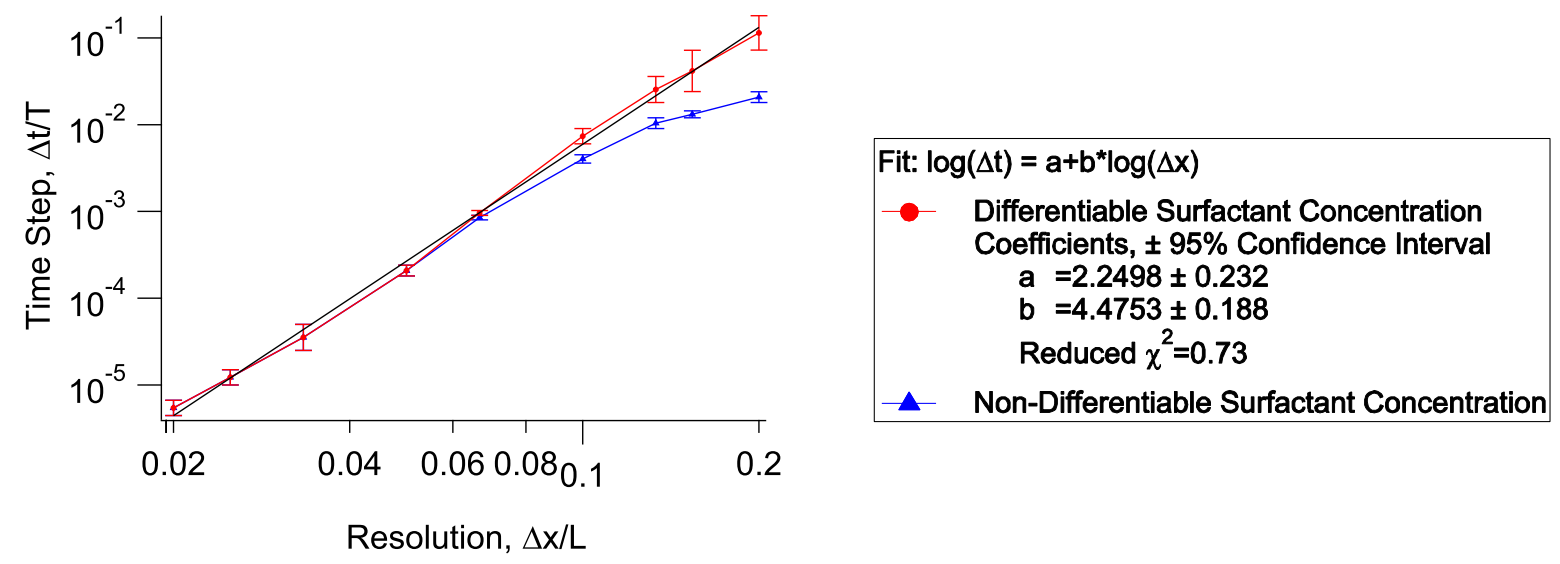

Figure 10: Maximum $\Delta t$ vs. $\Delta x$ for differentiable and non-differentiable outward spreading disks. For resolutions less than $\Delta x=0.05$, the two curves coincide.

has diffused over the surface, the difference in surfactant concentration between adjacent cells will decrease, and a larger time step may be sufficient.

\section{Conclusion}

The goal of this research was to quantify the error that is introduced by a spatial and temporal grid on the results of a simulation, as well as to identify conditions under which these errors are negligible. We found that, as an initial condition is rotated away from a line of symmetry associated with the spatial grid, more asymmetry is introduced into the solution. We also found that the rotational mismatch, or the degree to which the solution changes as the initial condition is rotated, increases as the angle of rotation moves further from 0 or 90 degrees. However, both the asymmetry and the rotational mismatch decrease as the spatial resolution is improved. Therefore, for any desired level of accuracy in the results, it is possible to choose a small enough spatial resolution to ensure grid effects are sufficiently small.

However, there is a large trade-off between the spatial resolution and the time required for a simulation to complete. If the length of the grid cells is decreased by a factor of two, then the time step used must decrease by nearly a factor of twenty. Fortunately, a variable time step will greatly improve the run time of the simulation; as the surfactant spreads over the surface of the film, the difference in surfactant concentration between adjacent cells decreases, and a larger time step could be used.

The numerical solver created by Claridge, Levy, and Wong can be used to solve a wide range of problems; it is especially well suited for nonlinear systems of partial differential equations of mixed type. The results of this study will assist future users of this numerical solver in choosing a spatial and temporal resolution that suits their needs. Users of this solver will be able to accurately balance the benefit of reducing asymmetry and rotational mismatch with the cost (in computational time) required to achieve a given level of accuracy.

\section{Acknowledgements}

We would like to thank our advisor, Prof. Rachel Levy, and Prof. Tom Witelski for their tremendous help. Thank you also to Dr. J. Claridge, J. Wong, Prof. M. Shearer, Prof. K. Daniels, and TUNL. Additionally, we acknowledge NSF-FRG-0968154 and Research Corp. award 19788. 


\section{References}

[1] M.S. Borgas and J.B. Grotberg. Monolayer flow on a thin film. J. Fluid Mech., 193:154-170, 1988.

[2] J. Claridge, R. Levy, and J. Wong. Nonlinear implicit PDE solvers: implementation techniques and a CLAWPACK library. preprint, 2013.

[3] D.W. Fallest, A.M. Lichtenberger, C.J. Fox, and K.E. Daniels. Fluorescent visualization of a spreading surfactant. New Journal of Physics, 12:073029, 2010.

[4] R.J. LeVeque. Finite Volume Methods for Hyperbolic Problems. Cambridge Texts in Applied Mathematics, Vol. 31. Cambridge University Press, 2002.

[5] R.J. Leveque, M.J. Berger, et al. Clawpack v.4.5.0, Sep. 2010. http://www. clawpack.org.

[6] E. Peterson. Flow of thin liquid films with surfactant: analysis, numerics, and experiment. PhD thesis, North Carolina State University, 2010. 